\title{
Prevalence of Serum Antibodies to TORCH Infections among the Women of Child Bearing Age Visiting National Public Health Laboratory, Teku
}

\author{
Binit Lamichhane ${ }^{1}$, Binita Pudasaini ${ }^{1}$, Bishnu Upadhyay ${ }^{2}$, Mohan Sharma ${ }^{1}$ and \\ Shyam Prasad Khanal ${ }^{2}$ \\ ${ }^{I}$ St. Xavier's College, Kathmandu, Nepal \\ ${ }^{2}$ National Public Health Laboratory, Kathmandu, Nepal \\ e-mail: binitlamichhane@gmail.com
}

\begin{abstract}
The primary infection of Toxoplasma gondii, Rubella virus, Cytomegalovirus (CMV) and Herpes simplex virus (HSV) abbreviated as TORCH, remain a major problem in the women of child bearing age in Nepal. The main aim of this study was to determine the seroprevalence of TORCH infections among the women of child bearing age visiting the National Public Health Laboratory (NPHL), Kathmandu. Serum samples collected from 302 patients were tested for TORCH infections by IgM Enzyme Linked Immunosorbent Assay (ELISA). The seropositivity rate was found to be $18.82 \%$ (54/287) for T. gondii, $10.07 \%$ (28/278) for Rubella, 16.49\% (46/279) for CMV and $23.34 \%$ (67/287) for HSV. The seropositivity rates in pregnant women were $17.92 \%(19 / 106)$ for $T$. gondii, $11.54 \%$ (12/104) for Rubella, 19.23\% (20/104) for CMV and 25\% (26/104) for HSV, the statistical association of TORCH infections with pregnancy was insignificant $(\mathrm{P}>0.05)$. Similarly, the seropositivity rates in women with bad obstetric history (BOH) were 17.84\% (43/241) for T. gondii, 11.06\% (26/235) for Rubella, 18.57\% (44/237) for CMV and $26.14 \%$ (63/241) for HSV. The statistical association of CMV and HSV with previous obstetric performance were significant $(\mathrm{P}>0.05)$ while that of $T$. gondii and Rubella were insignificant $(\mathrm{P}<0.05)$. The seropositivity rate was found to be highest for HSV infection (23.34\%) followed by T. gondii (18.82\%), Cytomegalovirus (16.49\%) and Rubella (10.07\%)
\end{abstract}

Key words: TORCH infection, IgM ELISA, pregnant women, women with BOH

\section{Introduction}

Toxoplasmosis is caused by a protozoan, Toxoplasma gondii. It is asymptomatic in healthy individual and if acquired during pregnancy, especially as a primary infection may damage to the fetus (Yasodhara et al. 20011). Although of little concern in normal immunologically competent hosts, $T$. gondii infections can be fatal when the patient's immune system is unable to control the infection. Such is the case of congenital toxoplasmosis when $T$. gondii crosses the placenta during a primary maternal infection and causes disseminated infection of the fetus (Luft $e t$ al. 1984). Rubella is caused by an RNA virus and transmitted through airway or direct contact. In most cases, the disease is asymptomatic or has minor flulike symptoms and generally is without sequelae ( $\mathrm{Su}$ \& Guo 2002). In pregnant women, however, rubella might cause multiple organ defects in fetuses, which is called congenital rubella syndrome (CRS) (Webester 1998). Cytomegalovirus (CMV) infection is the most important congenital infection globally (Gandhoke et al. 2009). Human CMV poses an important public health problem as it may cause serious morbidity and mortality in congenitally infected newborns and immunocomprimised patients, most notably transplant recipients and HIV infected persons (Chakravarti et al. 2009). The infected infants may suffer sensorineural hearing loss, ocular damage, or impairment of 
cognitive and motor function (Fowler et al. 1993). The spectrum of infections caused by Herpes simplex Virus Type 1 (HSV-1) and Herpes simplex Virus Type 2 (HSV-2) includes oropharyngeal, genital and ocular lesions, neonatal infections, central nervous system infections and multisystem infections in the immunocomprimised individuals (Fatahzadeh \& Schwartz 2007). HSV-1 is usually associated with primary infections of the orofacial area and latent infection of the trigeminal ganglion, while HSV-2 is usually associated with genital infections and latent infection in sacral ganglia (Whitley 2001).

TORCH infections are associated with recurrent abortion, intrauterine growth retardation, intrauterine death, preterm labour, early neonatal death and congenital malformations (Surpam et al.2006). Serologic testing of both mothers and neonates could be useful for detecting malformations (Golalipour et al. 2009). All antenatal cases with bad obstetric history should be routinely screened for TORCH complex as early diagnosis and appropriate intervention of the infection will help in proper management of these cases (Turbadkar et al. 2003).

In developing country like Nepal, where most of women belong to the low socio-economic group, may be exposed to one or more of the TORCH agents that may lead to serious congenital malformations. Therefore, appropriate intervention measures, early diagnosis and treatment of TORCH infection have to be carried out to reduce these infections during pregnancy and their effect on outcome of pregnancy.

\section{Methodology}

The study was conducted at the Immunology section of National Public Health Laboratory (NPHL), Kathmandu from March to August, 2010. A total of 302 female patients of child bearing age suspected of TORCH infections were recruited in this study. Informed verbal consent was taken from all the patients orally. Toxoplasma gondii, Rubella virus, CMV and HSV-II IgM antibodies were tested as per the instruction of the manufacturer (Human, Germany). Data were statistically analyzed using Chi-square test.

\section{Data collection}

Data were collected from each female patient of child bearing age by interview through a questionnaire.
Clinical history (name, age, sex, signs and symptoms, past history of TORCH test, marital status, pregnancy and number of miscarriage or stillbirth, if any) of the patients were collected.

\section{Specimen collection and storage}

Following aseptic precautions, blood specimens were collected by vein puncture from female patient of child bearing age and were kept in labelled, clean and dry test tubes. The blood samples in the tubes were allowed to clot for 30 minutes at room temperature. The samples were then centrifuged for serum separation. The separated serum was then kept in other labelled tubes. The sera were then refrigerated at $2-8^{\circ} \mathrm{C}$ until tested. Few serum specimens for TORCH tests referred from different hospitals situated at Kathmandu valley were also received directly at NPHL.

\section{Enzyme linked immunosorbent assay (ELISA) procedure}

ELISA techniques for the detection of $\operatorname{IgM}$ antibodies to TORCH agents (Toxoplasma, Rubella, Cytomegalovirus and Herpes simplex virus) were performed as per the instruction of the manufacturer (Human, Germany).

\section{Data analysis}

Data were analyzed using Chi-square test with 5\% level of significance.

\section{Results and Discussion}

In this study, out of 302 suspected patients, 54 women (18.82\%) among 287 suspected cases were positive to Toxoplasma (IgM); 28 women (10.07\%) among 278 cases were positive to Rubella (IgM); 46 women $(16.49 \%)$ among 279 suspected cases were positive to CMV (IgM) and 67 women $(23.34 \%$ ) among 287 suspected cases were positive to HSV (IgM).

The seroprevalence of HSV infection was found to be highest $(25 \%)$ compared to other TORCH agents. The seroprevalence of HSV was followed by Cytomegalovirus (19.23\%), Toxoplasma (17.92\%) and Rubella (11.54\%). HSV infection was found to be highest among the TORCH infections in patients with bad obstetric history $(\mathrm{BOH})$ followed by Cytomegalovirus, Toxoplasma and Rubella whose seropositivity were $26.14 \%, 18.57 \%, 17.84 \%$ and $11.06 \%$ respectively.

TORCH infections were associated with inadvertent outcomes like multiple abortions, sterility, congenital 
malformations, intrauterine foetal deaths, still births and other reproductive failures, especially when they were acquired during the first trimester of the pregnancy. The detection of IgM antibody against TORCH was the best approach for the identification of these infections (Li et al. 2009). In this study, highest IgM seropositivity was seen with HSV (23.34\%) followed by T. gondii (18.82\%), CMV (16.49\%) and Rubella (10.07\%). HSV (IgM) and Toxoplasma (IgM) was found to have higher seroprevalance because in addition to congenital transmission, HSV was transmitted sexually and T. gondii was transmitted orally by eating raw meat or exposure to infected cat faeces. Similarly, the lower seroprevalance of Rubella (IgM) could be due to naturally acquired immunity.
T. gondii is known as an etiological agent in recurrent pregnancy wastages and its IgM seropositivity rate in pregnant women was found to be $17.92 \%$ in our study. In a study done in India, it was found to be $13.1 \%$. Lower seroprevalance were found in studies done in other countries (Ribeiro et al. 2008, Alvarado-Esquivel et al. 2009). Among the women with $\mathrm{BOH}$, prevalence of IgM antibodies against $T$. gondii was found to be $17.84 \%$ which was in concordance with the finding of a comparable study $(15.43 \%)$ conducted earlier (Lamichhane et al. 2007). In a recent study in India, $21.9 \%$ of women with $\mathrm{BOH}$ were positive to $T$. gondii $\operatorname{IgM}$ antibodies (Sarkar et al. 2012).

Table 1. Correlation of TORCH infections with pregnancy

\begin{tabular}{|c|c|c|c|c|}
\hline Pregnancy & & \multicolumn{3}{|c|}{ Seropositivity percentage of } \\
\hline & Toxoplasma (IgM) & Rubella (IgM) & Cytomegaloviurs (IgM) & $\begin{array}{c}\text { He rpes Sirnplex } \\
\text { Virus (IgM) }\end{array}$ \\
\hline Pregnant & $17.92(19 / 106)$ & $11.54(12 / 104)$ & $19.23(20 / 104)$ & $25(26 / 104)$ \\
\hline $\begin{array}{l}\text { Non-Pregnant } \\
P \text { value }\end{array}$ & $19.34(35 / 181)$ & $9.2(16174)$ & $\begin{array}{l}14.86(261175) \\
P=0.05\end{array}$ & $22.40(41 / 183)$ \\
\hline
\end{tabular}

Rubella or German measles is an infectious disease caused by Rubella virus which can pass from the pregnant women's bloodstream to infect her unborn child causing congenital Rubella syndrome (Hirsch 2006). In the present study, Rubella IgM seropositivity rate of pregnant women was found to be $11.54 \%$. In a study done in Nigeria, $3.9 \%$ of pregnant women were positive to Rubella IgM and was found to be $7.7 \%$ in another study (Grace et al. 2009, Linguissi et al. 2012). Some recent studies reported lower seroprevalance of Rubella (Vilibic-Cavlek et al. 2011 Qin et al. 2011). In pregnant women, rubella might cause multiple organ defects in fetuses, which is called congenital rubella syndrome (CRS) (Webester 1998). In our study, Rubella IgM seropositivity in women with $\mathrm{BOH}$ was $11.06 \%$ which was higher than found in an earlier study in Nepal (4\%) (Lamichhane et al. 2007). In recent studies, Rubella in women with $\mathrm{BOH}$ were found to be $30.4 \%$ and $4.65 \%$ respectively (Sen et al. 2012, Sadik et al. 2012).

CMV infections were mostly inapparant, the virus could cause wide range of diseases in neonates (Kim 2010). The present study showed CMV IgM seropositive rate of $19.23 \%$ in the pregnant women. However, lower seroprevalance was found in other studies (Guo 1992, Chakravarty et al. 2005, Munro et al. 2005). In this study, CMV IgM seroprevalance was found to be $18.57 \%$ in $\mathrm{BOH}$ patients. Similar study done in Nepal showed 9.33\% (Lamichhane et al. 2007). In a study done in India recently, $34.7 \% \mathrm{BOH}$ patients were positive to CMV IgM (Sen et al. 2012) while other studies showed lower seroprevalence (Turbadkar et al. 2003, Sadik et al. 2012).

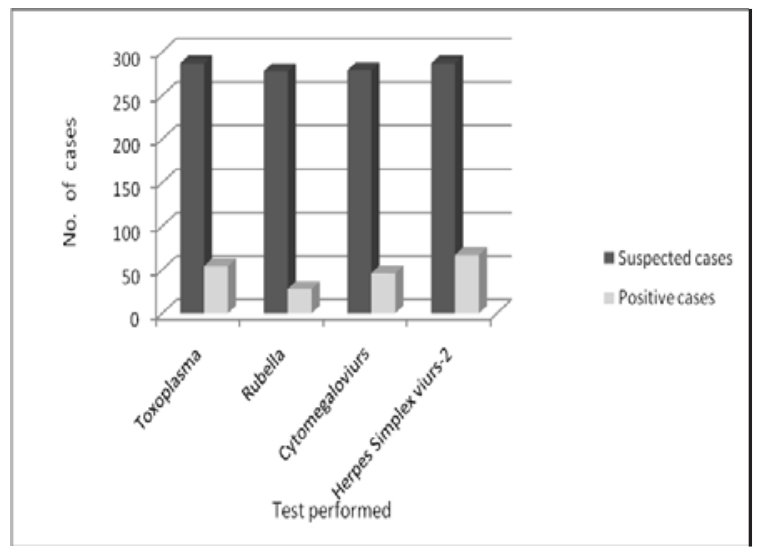

Fig 1. TORCH (IgM) test results

Likewise, our study showed that $25 \%$ of pregnant women were positive to HSV IgM antibodies. Studiesdone in Croatia and China have reported 
seropositivity rate of $1.2 \%$ and $2.45 \%$ respectively (Vilibic-Cavlek et al. 2011, Qin et al. 2011). Seropositivity rate of HSV IgM among the BOH patients of our study was $26.14 \%$. The seropositivity was found to be $11.33 \%$ in a previous study (Lamichhane et al. 2007). A study done in India showed higher seroprevalance of $33.5 \%$ (Sen et al. 2012). However, lower seroprevalance was reported in a study done in South India (Sadik et al. 2012).

This showed that the TORCH infections in the women of reproductive age group was mostly due to the infections which had very common route of transmissions in Nepal, for example, HSV was transmitted sexually and $T$. gondii was transmitted mainly from the food borne route. The low seroprevalance of Rubella might be because of the fact that it was self-limiting illness and provides lifelong natural immunity. The overall result emphasized the need for protection against these infections by safe sexual practice, maintenance of good hygiene and vaccination.

Table 2. Correlation of TORCH infection with previous obstetric performance

\begin{tabular}{|c|c|c|c|c|c|}
\hline \multirow{2}{*}{$\begin{array}{l}\text { Previous } \\
\text { performance }\end{array}$} & \multirow[t]{2}{*}{ obste tric } & \multicolumn{4}{|c|}{ Se ropositivity percentage of } \\
\hline & & Toxoplasma (IgM) & Rubella (IgM) & Cytornegaloviun (IgM) & $\begin{array}{c}\text { Herpes Simplex } \\
\text { Vinus (IgM) }\end{array}$ \\
\hline $\mathrm{BOH}$ & & $17.84(43 / 241)$ & $11.06(26 / 235)$ & $18.57(44 / 237)$ & $26.14(63 / 241)$ \\
\hline $\begin{array}{l}\text { Normal } \\
\text { performance }\end{array}$ & obstetric & $23.91(11 / 46)$ & $4.65(2 / 43)$ & $2.76(2 / 42)$ & $8.70(4 / 46)$ \\
\hline P value & & $P=0.05$ & & $P<0.05$ & \\
\hline
\end{tabular}

\section{References}

Alvarado-Esquivel, C., A. Torres-Castorena, O. Liesenfeld, C.R. Garcia-Lopez, S. Estrada-Martinez, A. SifuentesAlvarez, J.F. Marsal-Hernandez, R. Esquivel-Cruz, F. Sandoval-Herrera, J.A. Castaneda, and J.P. Dubey. 2009. Seroepidemiology of Toxoplasma gondii infection in pregnant women in rural Durango, Mexico. J. Parasitol. 95(2):271-274

Chakravarti, A., B. Kashyap and M. Matlani. 2009. Cytomegalovirus infection: an Indian perspective. Indian J. Med. Microbiol. 27(1):3-11.

Chakravarty, A., B Kashyap and K. Rathi. 2005. The sero epidemiological study on cytomegalovirus in women of child-bearing age with special reference to pregnancy and maternal-fetal transmission.Indian $J$. Pathol. Microbiol. 48(4):518-521.

Fatahzadeh, M. and R.A. Schwartz. 2007. Human herpes simplex virus infections: epidemiology, pathogenesis, symptomatology, diagnosis, and management. J. Am. Acad. Dermatol 57(5):737-763.

Fowler, K.B., S. Stagno and R.F. Pass. 1993. Maternal age and congenital cytomegalovirus infection: Screening of two diverse newborn populations: 1980-1990. J. Infect. Dis. 168:552-556.

Gandhoke, I.,R. Aggarwal, S.A. Hussain, S.T. Pasha, P. Sethi, S. Thakur, S. Lal and S. Khare. 2009. Congenital CMV infection; diagnosis in symptomatic infants. Indian J. Med. Microbiol 27(3):222-225.

Golalipour, M.J., B. Khodabakhshi and E. Ghaemi. 2009. Possible role of TORCH agents in congenital malformations in Gorgan, northern Islamic Republic of Iran. East Mediterr. Health J. 15(2):330-336.

Grace, P., A. Ginikanwa, A. Hannah, G. Sarah, A. Simon and F. Joseph. 2009. Serologic survey of specific rubella virus IgM in the sera of pregnant women in Makuridi, Benue State, Nigeria. Af.r J. Reprod. Health. 13(2):69-73.

Guo, T. 1992. Study of primary CMV infection in pregnant women. Zhonghua Liu XingBing Xue Za Zhi. 13(2):76-78. [In Chinese, English abstract]

Infections: Rubella (German measles). Hirsch, L. 2006: Available at: http://www.kidshealth.org/parent infections/Rubella [Date accessed: 15 September, 2011]

Kim, S. 2010. Congenital and perinatal cytomegalovirus infection. Korean J. Pediatr. 53(1):14-20.

Lamichhane, S., S. Malla, S. Basnyat, S. Khanal, S. Dumre, L. Maharjan and P. Shrestha. 2007. Seroprevalence of IgM antibodies against the agents of torch infections among the patients visiting National Public Health Laboratory, Teku, Kathmandu. J. Nepal Health Res. Counc. 5:21-25.

Li, Z., C. Yan, P. Liu, R. Yan and Z. Feng. 2009. The prevalence of the serum anti-bodies to TORCH among women before pregnancy or in the early period of pregnancy in Beijing. Clinica Chimica Acta 403: 212-15.

Linguissi, L.S., B.M. Nagalo, C. Bisseye, T.S. Kagoné, M. Sanou, I. Tao, V. Benao, J. Simporé and B. Koné. 2012. Seroprevalence of toxoplasmosis and rubella 
Binit Lamichhane et al/Prevalence of Serum Antibodies

in pregnant women attending antenatal private clinic at Ouagadougou, Burkina Faso. Asian Pac J. Trop. Med. 5(10):810-3.

Luft, B.J., R.G. Brooks, F.K. Conley, R.E. McCabe and J.S. Remington. 1984. Toxoplasmic encephalitis in patients with acquired immune deficiency syndrome. J. Am. Med. Assoc. 252(7):913-917.

Munro, S.C. , B. Hall, L.R. Whybin, L. Leader, P. Robertson, G.T. Maine and W.D. Rawlinson. 2005. Diagnosis of and screening for cytomegalovirus infection in pregnant women.J. Clin. Microbiol. 43(9):4713-4718.

Qin, W., D.C. Hu and H.L. Pang. 2011. Detection of serum IgM and IgG specific for TORCH in 1307 women in the period of pre-pregnancy and pregnancy. Zhonghua Shi Yan He Lin Chuang Bing Du Xue Za Zhi. 25(4):292-4. [In Chinese, English abstract]

Ribeiro, A.C., M.S. Mutis and O. Fernandes. 2008. Association of the presence of residual antiToxoplasma gondii IgM in pregnant women and their respective family groups in Miracema, Northwest Rio de Janeiro, Brazil. Memorias DoInstituto Oswaldo Cruz 103(6):591-4.

Sadik, M.S., H. Fatima, K. Jamil, and C. Patil. 2012. Study of TORCH profile in patients with bad obstetric history. Biology and Medicine 4(2):95-101.

Sarkar, M.D., B. Anuradha, N. Sharma and R.N. Roy. 2012. Seropositivity of toxoplasmosis in antenatal women with bad obstetric history in a tertiary-care hospital of Andhra Pradesh, India. J. Health Popul. Nutr. 30(1):87-92.
Sen, M.R., B.N. Shukla, and B. Tuhina. 2012. Prevalence of serum antibodies to TORCH infection in and around Varanasi, Northern India. J. Clin. Diagn. Res. 6(9): 1483-5.

Su, S.B. and H.R. Guo. 2002. Seroprevalance of rubella among women of childbearing age in Taiwan after nationwide vaccination. Am J. Trop. Med. Hyg. 67(5):549-553.

Surpam, R.B., U.P. Kamlakar, R.K. Khadse, M.S. Qazi andS.V. Jalgaonkar. 2006. Serological study for TORCH infections in women with bad obstetric history. Obstet. Gynecol. India. 56(1):41-43.

Turbadkar, D., M. Mathur and M. Rele. 2003. Seroprevalence of torch infection in bad obstetric history. Indian J. Med. Microbiol. 21(2):108-110.

Vilibic-Cavlek, T., S. Ljubin-Sternak, M. Ban, B. Kolaric , M. Sviben , and G. Mlinaric-Galinovic. 2011. Seroprevalance of TORCH infections in women of childbearing age in Croatia. J. Matern. Fetal. Neonatal. Med. 24(2):280-3.

Webester, W.S. 1998. Teratogen update: congenital rubella. Teratology 58(1):13-23.

Whitley, R.J. 2001. Herpes simplex viruses. In: Fields Virology $4^{\text {th }}$ Ed. (Eds. D.M. Knipe, and P.M. Howley ). Philadelphia, USA, Pp.2461-2509.

Yasodhara, P., B.A. Ramalakshmi, A.N. Naidu, and L. Raman. 2001. Prevalence of specific IgM due to Toxoplasma, Rubella, CMV and C. trachomatis infections during pregnancy. Indian J. Med. Microbiol. 19:52-56. 
Nepal Journal of Science and Technology Vol. 15, No.2 (2014) 85-90 